\title{
Head-up Tilt Test Combined With Isoproterenol Infusion Provokes Coronary Vasospastic Angina
}

\author{
Setsuya Miyata, MD; Hitoki Inoue, MD; Masashi Horimoto, MD; Satomi Hamasakai, MD; \\ Eiji Shibuya, MD; Haruhiko Yoshimura, MD; Satoshi Ohizumi, MD; \\ Hisashi Matsushima, MD; Keiichi Igarashi, MD; Takashi Takenaka, MD
}

\begin{abstract}
The association of the autonomic nervous system with coronary vasospasm has been controversial. The aim of the present study was to examine the involvement of the autonomic nervous system in coronary vasospasm by applying the head-up tilt (HUT) test to patients with coronary vasospastic angina. Fifteen consecutive patients with coronary vasospastic angina and without significant organic coronary stenoses underwent the HUT test. Prior to the test, coronary spasm was documented angiographically by using an intracoronary injection of acetylcholine or ergonovine. The HUT test was performed in the early morning and repeated in the afternoon if the test was positive in provoking angina pectoris and syncope or presyncope. If the test was negative, it was repeated under intravenous infusion of isoproterenol at a rate of $1-2 \mu \mathrm{g} / \mathrm{min}$. The HUT test under isoproterenol infusion in the morning provoked vasospastic angina with syncope or presyncope in 9 of the 15 patients. In the test-positive group, heart rate was significantly reduced $(104 \pm 17$ beats $/ \mathrm{min}$ to $84 \pm 25$ beats $/ \mathrm{min}, \mathrm{p}<0.05)$, which preceded a reduction in systolic blood pressure $(158 \pm 25 \mathrm{mmHg}$ to $125 \pm 17 \mathrm{mmHg}, \mathrm{p}<0.01)$, angina attack and syncope. The HUT test without isoproterenol infusion in the morning and the HUT test in the afternoon with or without isoproterenol infusion failed to provoke angina. The heart rate reduction preceding reduced systemic blood pressure and anginal attack suggested that parasympathetic nerve excitation plays an important role in coronary vasospasm. The results also implied that the HUT test combined with isoproterenol infusion is useful for the provocation of coronary spasm. (Jpn Circ J 1998; 62: 670-674)
\end{abstract}

Key Words: Head-up tilt test; Isoproterenol; Vasospastic angina

$\mathbf{T}$ he precise mechanism of coronary spasm remains ill defined. Earlier studies have shown that coronary spasm can be induced by stimulation of the autonomic nervous system ${ }^{1-4}$ However, it is unclear whether the sympathetic or parasympathetic nerve system plays a predominant role in coronary spasm. The head-up tilt (HUT) test has been commonly used for diagnosis of neurally mediated syncope, 5 the mechanism of which is considered to be a reflex vagal excitation preceded by sympathetic stimulation via mechanoreceptor C-fibers in the left ventricle, in the setting of excessively reduced venous return!-8 We performed the HUT test with patients who had vasospastic angina, postulating that vagal excitation, a final reflex pathway in the syncope during the test, may provoke coronary vasospasm.

\section{Patients}

The study included 15 consecutive Japanese patients (12 men, 3 women; age $60.9 \pm 8.5$ (mean $\pm \mathrm{SD}$ ) years, range; $39-73)$ with coronary vasospastic angina. The angina was confirmed by intracoronary injection of acetylcholine, or ergonovine when the acetylcholine test was negative. The study excluded patients with the following: (i) a prior history of syncope or presyncope that was related or unrelated to cardiac arrhythmia; (ii) hypertrophic cardiomyopathy, aortic stenosis or diabetes mellitus; and (iii) organic

(Received November 25, 1997; accepted May 6, 1998)

Division of Cardiology, Sapporo National Hospital, Sapporo, Japan

Mailing address: Hitoki Inoue, MD, Kikusui 4-2, Shiroishi-Ku, Sapporo 003, Japan coronary stenosis of more than $50 \%$.

\section{Cardiac Catheterization}

Medication was withdrawn from 4 days before coronary angiography. Following control coronary angiography, a provocation test for coronary spasm was done with a stepwise dose injection of acetylcholine into the left coronary artery $(20,50,100 \mu \mathrm{g})$ and subsequently into the right coronary artery $(20,50 \mu \mathrm{g})$. If coronary spasm was not provoked, ergonovine was injected in a stepwise dose (10, 20 and $30 \mu \mathrm{g}$ ) into the left and subsequently into the right coronary arteries. The test was defined as positive when intense coronary constriction or coronary occlusion was noted together with chest pain and an ST-segment shift on electrocardiogram (ECG). To identify organic coronary stenosis, isosorbide dinitrate of $2.5 \mathrm{mg}$ was finally injected into both coronary arteries. The angiographic findings in 15 patients are summarized in Table 1.

\section{The HUT Test}

After written informed consent was obtained from each patient, medication was withdrawn from 4 days before the HUT test. The test was conducted in the early morning after overnight fasting and repeated in the afternoon if the morning test was positive. The HUT test was performed within 2 weeks of documentation of coronary vasospasm. Following a $15 \mathrm{~min}$ rest in a supine position, patients were tilted upright to $80^{\circ}$ and positioned for $15 \mathrm{~min}$ using a supporting foot board9,10 Electrocardiograms and blood pressure in the radial artery were continuously recorded during the test. Drugs were injected through a cannula in a 
Table 1 Electrocardiographic and Coronary Arteriographic Findings in 15 Patients With Vasospastic Angina

\begin{tabular}{|c|c|c|c|c|c|}
\hline \multirow{2}{*}{ Patient no. } & \multirow{2}{*}{ Age (years) } & \multicolumn{4}{|c|}{ Angiography } \\
\hline & & Baseline & Stimulus & During spasm & ECG changes \\
\hline 1 & 63 & normal & Ach $(10 \mu \mathrm{g})$ & Seg. $7100 \%$ & $S T \uparrow$ in $V_{l-5}$ \\
\hline 2 & 68 & normal & Ach $(50 \mathrm{\mu} g)$ & Seg. $2100 \%$ & $S T \uparrow$ in $I I, I I I, a V_{F}$ \\
\hline 3 & 70 & normal & Ach (-) & Seg. $2100 \%$ & $S T \uparrow$ in $I I, I I I, a V_{F}$ \\
\hline 4 & 60 & normal & Ach $(20 \mu \mathrm{g})$ & Seg. $2100 \%$ & $S T \uparrow$ in $I I, I I I, a V_{F}$ \\
\hline 5 & 65 & normal & Ach $(20 \mu \mathrm{g})$ & Seg. $6100 \%$ & $S T \uparrow$ in $V_{l-5}$ \\
\hline 6 & 60 & Seg. $6 \quad 50 \%$ & Ach $(10 \mu \mathrm{g})$ & Seg. $7100 \%$ & $S T \uparrow$ in $V_{l-6}$ \\
\hline 7 & 52 & normal & Ach $(50 \mu \mathrm{g})$ & Seg. $6 \quad 90 \%$ & $S T \downarrow$ in $V_{3-6}$ \\
\hline 8 & 53 & normal & Ach $(50 \mu \mathrm{g})$ & Seg.13 100\% & $S T \uparrow$ in $I I, I I I, a V_{F}$ \\
\hline 9 & 39 & normal & Ach $(20 \mu \mathrm{g})$ & Seg $2100 \%$ & $S T \uparrow$ in $I I, I I I, a V_{F}$ \\
\hline 10 & 63 & normal & Ach $(20 \mu \mathrm{g})$ & Seg. $1100 \%$ & $S T \uparrow$ in $I I, I I I, a V_{F}$ \\
\hline 11 & 73 & normal & Ach $(20 \mu \mathrm{g})$ & Seg. $2100 \%$ & $S T \uparrow$ in $I I, I I I, a V_{F}$ \\
\hline 12 & 56 & normal & Ach $(20 \mu \mathrm{g})$ & Seg. $1100 \%$ & $S T \uparrow$ in $I I, I I I, a V_{F}, V_{5-6}$ \\
\hline 13 & 68 & normal & Ach $(20 \mu \mathrm{g})$ & Seg. $799 \%$ & $S T \uparrow$ in $V_{2-5}$ \\
\hline 14 & 59 & normal & $\operatorname{Ergo}(20 \mu \mathrm{g})$ & Seg. $1100 \%$ & $S T \uparrow$ in $I I, I I I, a V_{F}$ \\
\hline 15 & 65 & normal & Ach $(20 \mu \mathrm{g})$ & Seg. $1100 \%$ & $S T \uparrow$ in $I I, I I I, a V_{F}$ \\
\hline
\end{tabular}

Seg.1 to Seg.15 refers to the segments of the coronary ateries as defined by AHA Committee Report. 50\%, 50\% stenosis according to AHA Committee Report; ST $\uparrow, S T$ segment elevation; ST $\downarrow$, ST segment depression; Ach, acetylcholine; Ergo, ergometrine; Ach (-), spontaneous attack induced.

Table 2 Electrocardiographic Change and Angina Pain During Tilt Test With Isoproterenol Infusion in the Test Positive Patients

\begin{tabular}{clc}
\hline \hline \multirow{2}{*}{ Patient no. } & \multicolumn{2}{c}{ Head-up tilt test result } \\
\cline { 2 - 3 } & \multicolumn{1}{c}{ ECG changes } & Intensity of angina \\
\hline 1 & $S T \uparrow$ in $I, a V_{L}, V_{4-6}$ & 3 \\
2 & $S T \downarrow$ in II, $I I I, a V_{F}, V_{4-6}$ & 3 \\
3 & $S T \uparrow$ in II, III, aVF & 3 \\
4 & $S T \downarrow$ in II, III, aVF & 8 \\
5 & $S T \downarrow$ in $I, a V_{L}, V_{4-6}$ & 4 \\
6 & $S T \downarrow$ in $V_{4-6}$ & 3 \\
7 & $S T \downarrow$ in II,III, a $V_{F}, V_{4-6}$ & 7 \\
8 & $S T \uparrow$ in II,III, aVF & 2 \\
9 & $S T \downarrow$ in $V_{2-5}$ & 8
\end{tabular}

Patient nos. are identical to those in Table 1. ST $\uparrow, S T$ segment elevation; $S T \downarrow$, ST segment depression; maximum intensity of angina, 10.

peripheral vein. If the baseline HUT test was negative, the patients were returned to the supine position and underwent continuous isoproterenol infusion with an initial rate of 1-2 $\mu \mathrm{g} / \mathrm{min}$. When the heart rate had attained a $25 \%$ increment by a gradual elevation of the infusion rate, the HUT test was repeated. The test was considered positive if chest pain with electrocardiographic ST-segment changes was noted. The test was stopped when syncope, presyncope or chest pain with electrocardiographic changes occurred. Tilting time was within $20 \mathrm{~min}$ of when isoproterenol used.

\section{Statistical Analysis}

Data are shown as mean \pm SD. The Wilcoxon signedrank test was used to compare the paired data of blood pressure and heart rate before and after the HUT test. The Mann-Whitney U-test was used to compare blood pressure and heart rate between positive and negative patients for the HUT test. A p-value of $<0.05$ was considered statistically significant.

\section{Results}

All the HUT tests without isoproterenol infusion and the test with isoproterenol infusion in the afternoon failed to provoke angina in any of the patients. However, the HUT test combined with isoproterenol infusion in the morning
Table 3 Blood Pressure and Heart Rate During the Head-up Tilt Test. Comparison Between the Test-Positive and Test-Negative Groups

\begin{tabular}{ccccc}
\hline \hline & Baseline & HUT & ISO & ISO +HUT \\
\hline ISO + HUT positive group $(n=9)$ & & & \\
SBP $(\mathrm{mmHg})$ & $147 \pm 20$ & $141 \pm 20$ & $158 \pm 25$ & $125 \pm 17^{++}$ \\
DBP $(\mathrm{mmHg})$ & $68 \pm 9$ & $71 \pm 12$ & $60 \pm 11$ & $58 \pm 15$ \\
heart rate $($ bpm $)$ & $60 \pm 12$ & $72 \pm 19^{*}$ & $104 \pm 17$ & $84 \pm 25^{+}$ \\
ISO + HUT negative group $(n=6)$ & & & \\
SBP $($ mmHg $)$ & $132 \pm 26$ & $135 \pm 28$ & $138 \pm 17$ & $133 \pm 13$ \\
DBP $($ mmHg $)$ & $69 \pm 14$ & $78 \pm 17^{*}$ & $64 \pm 9$ & $72 \pm 10^{+}$ \\
heart rate $(\mathrm{bpm})$ & $73 \pm 16$ & $85 \pm 9$ & $115 \pm 6$ & $126 \pm 9$ \\
\hline
\end{tabular}

${ }^{*} p<0.05$ vs baseline $;{ }^{+} p<0.05$ versus $I S O ;{ }^{++} p<0.01$ vs ISO; SBP, systolic blood pressure; DPB, diastolic blood pressure; HUT, head-up tilt; ISO, isoproterenol; ISO + HUT, head-up tilt during isoproterenol infusion.

did provoke vasospastic angina with syncope or presyncope in 9 of the 15 patients (test-positive group in Table 2). Thus, it was noted that there was a diurnal variation of the occurrence of vasospastic angina provoked by the HUT test with isoproterenol infusion. In the positive group for the test, the time from the beginning of the HUT to the onset of syncope or presyncope was $534 \pm 210 \mathrm{sec}$. Six test-negative patients showed neither chest pain nor a syncopal event.

A representative ECG of the test-positive patient is shown in Fig 1 (patient No 3 in Tables 1 and 2). STsegment elevation in the inferior leads and a reduction in systemic blood pressure were found before the onset of syncope. Provoked angina was relieved in 7 patients by returning to a supine position from the tilted position. In 2 patients (Nos 3 and 9), however, angina was not easily relieved by the position change, and required $2.5 \mathrm{mg}$ of intravenous isosorbide dinitrate. Patients nos 2, 4, 5, 6 and 9 showed electrocardiographic ST-segment depression during the HUT test under isoproterenol infusion, whereas they had shown ST-segment elevation with intracoronary acetylcholine or ergonovine. In 6 of the 9 test-positive patients, the ST-segment changes during the HUT test were identical to those found in spontaneous anginal attacks.

The age of the patients was similar between the positive and negative groups for the HUT test $(64.0 \pm 6.1$ and $58.9 \pm 9.6$ years, $p=0.19$ ). Two of the 9 patients in the test- 
A

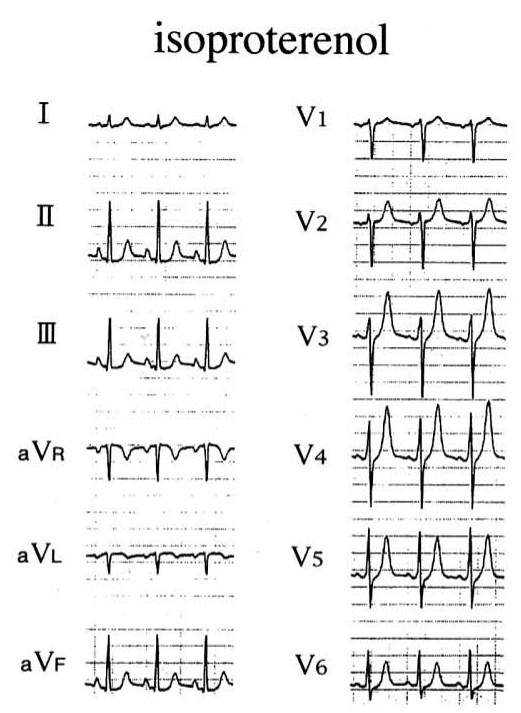

$\mathrm{BP}(\mathrm{mmHg})$

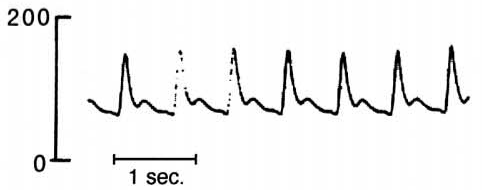

B
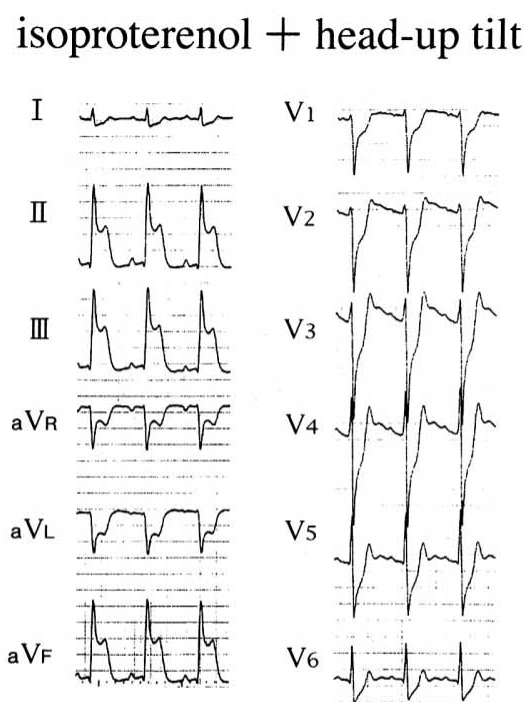

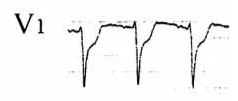

V2 y-yr

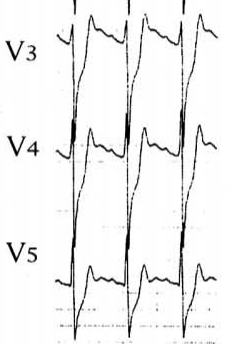

V6 $\sqrt{\min \mid \sin }$

$\mathrm{BP}(\mathrm{mmHg})$

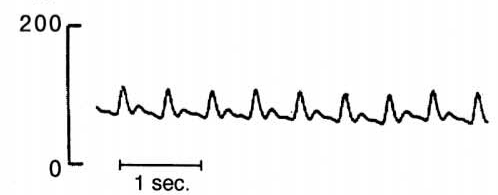

Fig 1. Representative recording of electrocardiograms and systemic blood pressure (BP) in a patient who developed syncope and vasospastic angina during head-up tilt under isoproterenol infusion. (A) An electrocardiogram taken in a supine position under isoproterenol infusion. (B) During the head-up tilt test under isoproterenol infusion ST-segment elevation in the inferior leads and reciprocal ST-segment depression in the precordial leads were noted with reduced heart rate and blood pressure.

positive group and 1 of the 6 patients in the test-negative group were female, showing no gender difference in the positive response to the test. At baseline before tilting, blood pressure and heart rate did not differ significantly between the 2 groups (Table 3). During the HUT test without isoproterenol infusion, diastolic blood pressure increased in the test-negative group $(69 \pm 14 \mathrm{mmHg}$ to $78 \pm 17 \mathrm{mmHg}, \mathrm{p}<0.05)$, but it remained unchanged in the test-positive group. Whereas heart rate increased in the test-positive group $(60 \pm 12$ beats/min to $72 \pm 19$ beats/min, $\mathrm{p}<0.05)$, it did not significantly increase in the test-negative group. During isoproterenol infusion before tilting, heart rate and blood pressure reached similar values in the 2 groups. With tilting under isoproterenol infusion, both systolic blood pressure $(158 \pm 25 \mathrm{mmHg}$ to $125 \pm 17 \mathrm{mmHg}$, $\mathrm{p}<0.01)$ and heart rate $(104 \pm 17$ beats/min to $84 \pm 25$ beats/min, $\mathrm{p}<0.05)$ were significantly reduced in the testpositive group but not in the test-negative group. The heart rate reduction preceded an angina attack, a reduction of blood pressure and syncope or presyncope. Angina attack, blood pressure reduction and syncope or presyncope occurred almost simultaneously.

\section{Discussion}

We considered that angina pectoris provoked by the HUT test under isoproterenol infusion was due to coronary spasm, because none of the patients had significant organic stenoses of the coronary arteries and the leads of ECGs of myocardial ischemia occurring during the test resembled those of a spontaneous anginal attack or of coronary spasm provoked with acetylcholine or ergonovine. Moreover, hypotension during the HUT test under isoproterenol infusion was not so pronounced as to cause reduced coronary perfusion pressure that would develop into myocardial ischemia.

\section{Mechanism of Coronary Spasm Induced by the HUT Com- bined With Isoproterenol Infusion}

The HUT test elicits neurally mediated syncope via the following chain of events, $6,7,11,12$ An excessive venous pooling secondary to head-up tilting leads to a decrease in ventricular preload, 6 , which reflexly stimulates the sympathetic nerve and produces a pronounced inotropic effect on the myocardium.1 ${ }^{1}$ This hypercontractile state has been evidenced echocardiographically as an increase in ventricular fractional shortening, a decrease in the end-systolic volume and an increase in intraventricular pressure ${ }^{12}$ These changes paradoxically excite cardiac mechanoreceptor Cfibers that are primarily stimulated by stretching of the cardiac muscles. The efferent reflex arc of the neurally mediated syncope is not well understood. The afferent neural traffic stimulates parasympathetic nuclei in the medulla (ie the nucleus ambiguous and the dorsal motor nucleus of the vagus nerve) and consequently augments parasympathetic activity. On the other hand, the afferent neural traffic suppresses the sympathetic nuclei in the rostral ventromedial and ventrolateral medulla? The excitation of the vagal efferent nerve and the suppression of sympathetic tone cause bradycardia and hypotension, respectively, and finally lead to syncope. A concomitant use of isoproterenol with the HUT test is known to augment the sympathetic nerve excitation at the entrance of the neural reflex arc during the test and eventually 
increases the sensitivity of the test for diagnosis of neurally mediated syncope $!^{3}$

Our study showed that a reduction in heart rate preceded the onset of provoked vasospastic angina with concurrent hypotension, which suggested that coronary spasm was likely to be caused by parasympathetic excitation. The study also demonstrated that coronary vasospasm was not induced by the HUT test alone or by isoproterenol infusion alone, but by their combination. This suggests that strong sympathetic excitation by isoproterenol infusion in advance of the HUT is necessary before the parasympathetic excitation that leads to coronary vasospasm.

In the present study, blood pressure and heart rate at syncope or presyncope were reduced significantly but not greatly. The intensity of the reductions in systemic blood pressure and heart rate seems insufficient to cause syncope or presyncope. The likely reason is that before sufficient reductions in heart rate and blood pressure were noted, the HUT with isoproterenol infusion had to be discontinued because of persistent chest pain with electrocardiographic ST-segment changes and syncope or presyncope.

Parasympathetic or Sympathetic Autonomic Nervous System: Which Plays a Predominant Role in Coronary Spasm?

Although the autonomic nerve system has been shown to play an important role in the mechanism of vasospastic angina, ${ }^{1-4}$ results are conflicting. Coronary spasm can be caused by an intracoronary injection of acetylcholine and prevented by premedication of atropine in some patients with vasospastic angina, 1,2 which suggests that the parasympathetic nervous system plays an important role in the pathogenesis of vasospastic angina. In contrast, some reports suggest a sympathetic nerve association with vasospastic angina: alpha-adrenergic stimulation by epinephrine $^{3}$ or exercise ${ }^{4}$ could induce coronary vasospasm. Further, a recent study based on the power spectrum analysis of heart rate variability emphasized the importance of sympathetic nerve excitation in vasospastic angina14,15 The low-frequency band of both R-R interval variability and systolic arterial pressure variability were greater in patients with vasospastic angina than in normal subjects when compared at 3 divided intervals in a day, suggesting sympathetic hyperactivity in patients with vasospastic angina ${ }^{14}$ Similarly, in patients with multivessel coronary spasm as compared with patients with single vessel coronary spasm, parasympathetic activity decreased and sympathetic activity increased 15 These controversies seem to be partly attributable to the difference in the provocation methods for coronary vasospasm. Most previous methods used neurotransmitters such as epinephrine and acetylcholine and did not stimulate autonomic nerves through the central nervous system. Moreover, in the study of heart rate variability, autonomic nerve activity was analyzed as a circadian variation but not examined immediately before or during an angina attack ${ }^{14}$

The HUT test with isoproterenol infusion enabled us to provoke vasospastic angina through reflex autonomic nerve excitation via the central nervous system. The angina was associated with hypotension and syncope, and was preceded by a reduction in heart rate. Thus, we concluded that coronary spasm is predominantly caused by parasympathetic nerve excitation, although it is possibile that strong sympathetic excitation is primarily needed in advance of the parasympathetic excitation.
Clinical Significance of the Present Study

The present study demonstrated that $60 \%$ of patients with vasospastic angina were positive for the HUT test under isoproterenol infusion in the morning. As none of the patients had a prior history of syncope or presyncope, the result does not indicate a high susceptibility to syncope in the test-positive group. The test can detect patients with vasospastic angina, where the parasympathetic nervous system plays a predominant role in the potentiation of vasospasm. Our study also demonstrated that the test is useful for the provocation of coronary spasm.

\section{Limitations and Consideration for Future Study}

Parasympathetic nerve excitation was considered to be responsible for coronary spasm in our study. To further delineate the parasympathetic nerve association with coronary spasm, premedication with atropine as an anticholinergic drug will be needed with the HUT test. In addition, heart rate variability immediately before and during the vasospastic angina provoked during the test remains to be analyzed.

\section{Conclusion}

The present study demonstrated that the HUT test with isoproterenol infusion provoked coronary spasm with syncope or presyncope in $60 \%$ of patients with vasospastic angina. Vasospastic angina and syncope with a reduction in systemic blood pressure were preceded by a reduction in heart rate. The finding suggests that parasympathetic nerve activation plays an important role in the provocation of coronary spasm in a subgroup of patients with vasospastic angina.

\section{Acknowledgments}

We thank Professor Kenjirou Kikuchi of the Asahikawa Medical College for his valuable advice.

\section{References}

1. Yasue H, Horio Y, Nakamura N, Fujii H, Imoto N, Sonoda R, et al: Induction of coronary artery spasm by acetylcholine in patients with variant angina: possible role of the parasympathetic nervous system in the pathogenesis of coronary artery spasm. Circulation 1986; 74: 955-963

2. Mikawa K, Goto M, Lee JD, Matsuyama F, Shimizu H, Kato T, et al: Super sensitivity of coronary arteries in variant angina to spasm induced by intracoronary acetylcholine. Am J Cardiol 1988; 61: $77-$ 82

3. Yasue H, Touyama M, Kato H, Tanaka S, Akiyama F: Prinzmetal's variant form of angina as a manifestation of alpha-adrenergic receptor mediated coronary artery spasm: documentation by coronary arteriography. Am Heart J 1976; 91: 148-155

4. Yasue H, Omote S, Takizawa A, Nagao M, Miwa K, Tanaka S: Circadian variation of exercise capacity in patients with Prinzmetal's variant angina: role of exercise-induced coronary arterial spasm. Circulation 1979; 59: $938-948$

5. Kenny RA, Ingram A, Bayliss J, Sutton R: Head-up tilt: a useful test for investigating unexplained syncope. Lancet 1986; 1: 1352-1355

6. Samoil D, Grubb BP: Vasovagal (neurally mediated) syncope: pathophysiology, diagnosis, and therapeutic approach. Eur J Cardiac Pacing Electrophysiol 1992; 4: 234-241

7. Rea R: Neurally mediated hypotension and bradycardia: Which nerve? How mediated? J Am Coll Cardiol 1980; 14: 1633-1634

8. Chen MY, Goldenberg F, Milstein S, Buetikofer J, Almquist A, Lesser J, et al: Cardiac electrophysiologic and hemodynamic correlates of neurally mediated syncope. Am J Cardiol 1989; 63: 66-72

9. Kapoor WN, Brant N: Evaluation of syncope by upright tilt testing with isoproterenol. Ann Intern Med 1992; 116: 358-363

10. Kosinski DJ, Grubb BP: Neurally mediated syncope with an update on indications and usefulness of head-upright tilt table testing and 
pharmacological therapy. Curr Opin Cardiol 1994; 9: 53-64

11. Sra J, Jazayeri M, Murthy V, Tchou P. Yue-Hua S, Troup P, et al: Sequential catecholamine changes during upright tilt: Possible hormonal mechanism responsible for pathogenesis of neurocardiogenic syncope (Abstract). J Am Coll Cardiol 1991; 17: 216A

12. Shalev Y, Gal R, Tchou P, Anderson AJ, Avitall B, Akhtar M, et al: Echocardiographic demonstration of decreased left ventricular dimensions and vigorous myocardial contraction during syncope induced by head upright tilt. J Am Coll Cardiol 1991; 18: 746-751

13. Kapor WN, Brant N: Evaluation of syncope by upright Tilt testing with isoproterenol. A nonspecific test. Ann Intern Med 1992; 116: $358-363$

14. Yamasaki F, Sato T, Takata J, Chikamori T, Ozawa T, Sasaki M, et al: Sympathetic hyperactivity in patients with vasospastic angina: Assessment by spectral analysis of heart rate and arterial pressure variabilities. Jpn Circ J 1996; 60: 10-16

15. Tsuchiya T, Okumura K, Yasue H, Kugiyama K, Ogawa H: Heart rate period variability in patients with variant angina. Am J Cardiol 1996; 77: 932-936. 